\title{
Plato und die Medizin seiner Zeit
}

\author{
O. Krückmann zugeeignet
}

Von K. AbeL

In diesen Zeilen sei der Versuch unternommen, Platos Stellung zur Medizin seiner Zeit in einigen bedeutsamen Punkten zu umreißen. Es ist von einem eigentümlichen Reiz, zu verfolgen, wie in einem der größten Geister der Antike, ja sogar des Menschengeschlechtes, sich gewisse Grundfragen der medizinischen $W$ issenschaft gespiegelt haben und aus welchen geschichtlichen Voraussetzungen sich seine Lösungsversuche erklären. Außerdem hat der Platonische Timaios, der den Stoff für unsere Untersuchung bietet, wie kaum ein zweites Werk den Gang der antiken Geistesgeschichte beeinflußt, so daß von hier aus Licht auf weite Strecken späterer Entwicklung fällt.

\section{a) Die Stellung des Timaios in Platos geistiger Entwicklung}

Der philologischen Forschung ist es in mühsamer Kleinarbeit gelungen, den umfänglichen literarischen Nachlaß Platos in vier Gruppen zu ordnen und den verschiedenen Lebensabschnitten des Philosophen zuzuweisen. Der Timaios ist entstanden, als Plato die Mittagshöhe des Lebens bereits überschritten hatte und der langsam heraufdämmernde Lebensabend seine Schatten vorauszuwerfen begann. Zusammen mit den Gesetzen darf er als das repräsentative Alterswerk des Philosophen gelten, wenn auch die Versuche, ihn einem bestimmten Jahr zuzuteilen, bislang zu keinem allgemein anerkannten Ergebnis geführt haben. Viele Jahre waren dahingegangen, seitdem Plato, der Sprößling eines vornehmen athenischen Hauses, dem einfachen Handwerker SoKrates begegnet war und unter seinem Einfluß die entscheidende Wendung seines inneren und äußeren Lebens erfahren hatte. Im Gastmahl hat er durch den Mund des genialen Alkibiades, dessen von selbstsüchtigen Motiven bestimmte Politik im Peloponnesischen Krieg seine Vaterstadt an den Rand des Abgrunds brachte, in Worte zu fassen versucht, was das Geheimnis und den Zauber der Persönlichkeit des verehrten Meisters ausmachte ${ }^{1}$. Der Waldschrat Sokrates, der unter einem Äußeren von grotesker Häßlichkeit wundersame Schätze des Geistes in

1 Symposion 215 A ff. 
seinem Gemüte barg, hatte den Jüngling Plato gefangengenommen und in seinen Bann gezogen. Unter dem Eindruck seiner Worte hat er hochfliegenden Künstlerträumen entsagt, den Gedanken politischer Betätigung zum Wohl seiner heißgeliebten Vaterstadt aufgegeben und statt dessen die Philosophie zum ausschließlichen Inhalt seines Lebens erhoben. Sie war für ihn keine müßige Spielerei mit Gedanken und Begriffen, sondern er sah in ihr ein zentrales Anliegen des geistigen Menschen, der zum eigenen und fremden Heil um den Sinn des Lebens ringt und im Besitz der Erkenntnis um das wahre Lebensziel seinem Dasein entsprechende Gestalt zu geben bemüht ist. Zu Lebzeiten des Lehrers hatte Plato geschwiegen; nach dessen Hinrichtung durch den Schierlingsbecher begann er mit einer Reihe von Werken an die Öffentlichkeit zu treten, obschon er die Überzeugung hegte, daß das geschriebene Wort neben der lebendigen Rede des mündlichen Lehrvortrags und Lehrgesprächs nicht bestehen könne. Zunächst wandelt er im engen Anschluß auf den Spuren des Meisters. Fragestellung und Lösungsverfahren tragen das Gepräge sokratischen Geistes: Es geht um gewisse ethische Grundwerte, deren Wesen man durch Aufstellung von Definitionen zu ergreifen bestrebt ist. Meist endet die Unterhaltung mit einer Aporie. Eben hierin enthüllt sich der eigentliche Sinn dieser Dialoge. Sie wollen aufrüttelnde Mahnrufe zu sittlicher Selbstbesinnung sein und den Leser zu ernsthaftem Nachdenken über die Grundlagen und Grundfragen der sittlichen Vernunft veranlassen.

Die darauf folgende Übergangszeit zeigt uns Plato auf dem Wege zu sich selbst. Im Menon vor allem ist mit der Entdeckung des von aller Erfahrung unabhängigen Gegenstandsbereiches der Mathematik ein denkwürdiger Schritt auf dem Weg zur Ideenlehre vollzogen. Plato glaubt aus der Eigentümlichkeit apriorischen Wissens schließen zu dürfen, daß der Menschenseele vor ihrer irdischen Existenz ein Dasein in überhimmlischen Gefilden beschieden war, an das sie in der apriorischen Erkenntnis die Erinnerung bewahrt hat ${ }^{2}$. Mit dem Gastmahl kommt die Ideenlehre zu vollem Durchbruch, die Überzeugung, daß es jenseits von Zeit und Raum eine Welt der ewigen Formen gibt, die in steter Gleichheit mit sich selbst verharren und sich dem Menschen wohl im Denken, nicht aber in der sinnlichen Wahrnehmung erschließen ${ }^{3}$. Im Staat fügt dann der Philosoph die Ergebnisse seiner ethischen und metaphysischen, politischen und erkenntnistheoreti-

${ }^{2}$ Menon $85 \mathrm{~B}$ ff.

${ }^{3}$ Symposion $210 \mathrm{E} \mathrm{ff}$. 
schen Spekulation zu einer großartigen Einheit zusammen und schafft damit das monumentale Werk seiner Reifezeit. Mit dem sich nähernden Alter drängen sich die ontologischen und logischen Aspekte seines Philosophierens in den Vordergrund. Mit dem Timaios wagt er sich in ein Gebiet, an dem er bisher vorbeigegangen war in dem Glauben, daß es keine sichere Erkenntnis gestatte, in das Reich des Werdens und der Veränderung. Zwar war er in dieser Überzeugung nicht wankend geworden, doch drängte es ihn, die bunte Fülle der Erscheinungen, die sich ihm in der Erfahrungswelt darboten, von dem in jahrelanger, zäher philosophischer Denkarbeit errungenen Standpunkt aus zu meistern. Ausklang seines Werkes bilden die Gesetze, in denen der Versuch unternommen wird, das erhabene Staatsideal der Politeia den tatsächlichen Gegebenheiten der Welt, in die wir gestellt sind, anzunähern.

\section{b) Die medizinischen Abschnitte im Aufbau des «Timaios»}

Der Timaios bildet das Anfangsstück einer Trilogie oder vielleicht auch Tetralogie. Der schriftstellerische Plan, dem er sein Entstehen verdankt, ist nicht zu Ende gereift. Im gegenwärtigen Zusammenhang ist es müßig, zu erörtern, ob die Kräfte des alternden Denkers bei der Ausführung seines Vorhabens" erlahmten oder ob die Einsicht in das Untunliche des Unterfangens zur Aufgabe der ursprünglichen Absicht Anlaß gab. Auch ob das Fallenlassen des Urplans Umarbeitungen im vollendeten Anfangsteil zur Folge hatte, mag dahingestellt bleiben. Wir halten uns an das fertiggestellte Werk und versuchen zunächst einen Überblick über seinen gedanklichen Aufbau zu gewinnen, um Klarheit zu erhalten, von welchen allgemeinen Voraussetzungen ausgehend Plato seine medizinischen Anschauungen entwickelt. Das Gespräch setzt ein mit einem Rückgriff auf eine fiktive Unterhaltung am Vortage über das Wesen des besten Staates, in der wichtige Bestandstücke des ideellen Gehalts der Platonischen Politeia ins Gedächtnis zurückgerufen werden ${ }^{4}$. An der Unterredung beteiligen sich außer Sokrates Platos Onkel Kritias, der Pythagoreer Timaios aus Lokroi, einer Stadt Unteritaliens, und Hermokrates, der syrakusanische Staatsmann, der seine Mitbürger zum energischen Widerstand gegen den athenischen Expansionsdrang des sizilischen Abenteuers (415-413) aufrief. Daran anschließend berichtet Kritias die halbverschollene Sage von dem Wunderland Atlantis jenseits der Säulen des Herakles, das einer gewaltigen Erdkatastrophe zum

4 Timaios $17 \mathrm{C} \mathrm{ff}$. 
Opfer fiel ${ }^{5}$. Dort war das staatliche Leben in Formen organisiert, wie sie der Platonische Staat als gültiges und verpflichtendes Vorbild aufzurichten strebte. Dann erteilt man das Wort Timaios, damit er seine Theorie der Weltentstehung entwerfe ${ }^{6}$. Man hat gemeint, Plato benutze Timaios nicht als Sprachrohr seines eigenen philosophischen Glaubensbekenntnisses, sondern mache ihn zum Verkünder streng altpythagoreischer Lehre, entsprechend den Gesetzen historischer Wahrheit. Kritischer Nachprüfung hält eine solche Ansicht nicht stand. Der Timaios ist durchsetzt mit Gedankengut, das uns aus anderen Schriften des Philosophen wohl vertraut ist. Für seine Ausführungen erhebt Timaios keinen Anspruch auf Wahrheit, sondern nur auf Wahrscheinlichkeit? ${ }^{\text {? }}$ Sie erstrecken sich auf die Welt des Werdens, in der zwar die Ideen als richtunggebende Kräfte einstrahlen, jedoch keine volle Herrschaft über das stoffliche Prinzip erlangen, so daß die Dinge der Sinnenwelt dem Gesetz ständigen Wechsels unterliegen. Mit der Unbeständigkeit des Erkenntnisobjektes ist jedoch auch die Unsicherheit der Erkenntnis gegeben. Der Gang der Darstellung spiegelt keineswegs den Verlauf der Weltschöpfung. Denn die Zeit ist durchaus nicht als eine Ordnungsform gefaßt, die allem Werden vorausliegt und sich allem Geschehen als unentrinnbares Gesetz aufzwingt, sondern als ein schwaches Abbild der Ewigkeit und Anhängsel der kreisenden Bewegungen, welche die Himmelskörper in ewigem Gleichmaß vollziehen ${ }^{8}$. Der göttliche Werkmeister, der Demiurg - eine Vorstellung, die uns bei Plato erstmals in der Geschichte der griechischen philosophischen und theologischen Spekulation entgegentritt -, hat das Weltall im Hinblick auf die Ideen geschaffen als ein einziges, riesiges, beseeltes, kugelgestaltiges Lebewesen von göttlichem Rang und höchstmöglicher Vollendung. Absolute Vollendung war dem Werk des Weltenschöpfers versagt, da es nicht nur geistgezeugt, sondern auch stoffgeboren ist. Das Stoffprinzip der Ananke, das sich in den vier empedokleischen Elementen, Feuer, Wasser, Luft und Erde, entfaltet, errichtet vor der reinen Verwirklichung der Vernunftzwecke gewisse unübersteigbare Schranken. Für die Unvollkommenheiten, die so in den Weltbau Eingang finden, ist der göttliche Werkmeister nicht verantwortlich. Im schroffen Gegensatz zu homerischer Vorstellungsweise verkündet Plato das Dogma, daß die Gottheit über das schwanke Hin und Her, das jähe

5 Timaios $21 \mathrm{E} \mathrm{ff.}$

${ }^{6}$ Timaios $27 \mathrm{~B}$ ff.

7 Timaios $27 \mathrm{D}$ ff.

8 Timaios $37 \mathrm{D}$ ff. 
Auf und $\mathrm{Ab}$ der Gefühlswallungen erhoben und erhaben ist ${ }^{9}$. So war das Streben des göttlichen Weltenbaumeisters auf das Beste gerichtet, und nicht aus scheelsüchtigem Neid hat er sich mit der Schaffung des Mindervollendeten zufriedengegeben. An die Schaffung des Makrokosmos schließt sich die des Mikrokosmos, des Menschen, in ausgeprägter Parallelität ${ }^{10}$. Die Vernunftseele, das herrschende Prinzip in der menschlichen Natur, ist aus den formenden Händen des göttlichen Weltenbildners selbst hervorgegangen und mit Unsterblichkeit begabt. Die Bildung des Leibes aus den vier Elementen und die Einpflanzung des Geistes in seine irdische Behausung überließ er den niederen Göttern. Das medizinische Wissen seiner Zeit über die Einrichtung des menschlichen Körpers und die Eigenart der in ihm sich abspielenden Lebensvorgänge sucht Plato in überaus geistreicher Weise vom Standpunkt seiner Seinslehre dem Verständnis zu erschließen. Bei der zentralen Bedeutung, welche das Seelisch-Geistige in Platos Weltanschauung besitzt, ist es durchaus gerechtfertigt, mit der Betrachtung der Verschmelzung von Seele und Leib einzusetzen und zunächst in den Hauptzügen eine Geschichte des Problems vom Sitz der Seele bis auf Plato zu entwerfen.

\section{c) Seelensitz}

In der zweiten Hälfte des sechsten vorchristlichen Jahrhunderts wirkte in der unteritalischen Stadt Kroton der von pythagoreischem Denken tief beeinflußte Arzt Alkmaion. Als Wegbereiter der wissenschaftlichen Anatomie hat er sich einen Ehrenplatz in der Geschichte der Medizin gesichert. Er ist der erste, von dem uns überliefert wird, daß er an Tieren Sektionen und sogar Vivisektionen vorgenommen hat ${ }^{11}$. Ob er darüber hinaus die menschliche Leiche zum Gegenstand seiner anatomischen Studien gemacht hat, darüber liegen verläßliche Zeugnisse nicht vor, und es finden sich keinerlei Anhaltspunkte, die zu einem solchen Schluß nötigten oder auch nur berechtigten. Bei der Untersuchung der Sinneswerkzeuge wurde Alkmaion bald inne, daß die Organe des Gesichts- und Gehörs-, des Geruchs- und des Geschmackssinnes - über den Tastsinn äußerte er sich nicht - in enger Verbindung mit dem Gehirn stehen ${ }^{12}$. Ein besonders ausführlicher Bericht, der

${ }^{9}$ Timaios 29 D f; vgl. Phaidros 247 A.

10 Timaios $42 \mathrm{E} \mathrm{ff}$.

${ }^{11}$ Vorsokratiker $I^{6}, 212,23$; vgl. 213, 31 u. a. (Diels-Kranz).

12 Vorsokratiker $I^{6}, 212,6 \mathrm{ff}$. (Drels-KraNz). 
einem spätantiken Kommentar zu Platons Timaios entstammt, liegt über die Augenanatomie vor mit eingehender Beschreibung des N. opticus ${ }^{13}$. Da hier Alkmaions Ergebnisse mit denen seiner Nachfolger, des AristotelesSchülers Kallisthenes und des Anatomieprofessors am Museion zu Alexandreia, Herophilos von Chalkedon, zusammengearbeitet sind, ist es leider äußerst schwierig, das spezifisch Alkmaionische Erkenntnisgut herauszulösen und anzugeben, wie weit der Krotoniate sich Einsicht in den Bau des Auges erworben hatte. Aber auch ohne dies darf die Behauptung aufgestellt werden, daß die angelegentliche Beschäftigung mit dem anatomischen Bau der Sinnesorgane dem unteritalischen Arzt die Erkenntnis schenkte, daß das Gehirn als Sitz der Denktätigkeit zu gelten habe ${ }^{14}$. Man findet zwar gelegentlich die Ansicht vertreten, daß Alkmaion zu seiner grundlegenden Erkenntnis auf spekulativem Wege gelangt sei, daß er sich habe leiten lassen von der Pythagoreischen Vorstellung, die Kugel sei das vollendetste stereometrische Gebilde, und darum den kugelförmigen Schädel als den würdigsten Körperteil für die Beherbergung der spezifisch menschlichen Erkenntnisfähigkeit angesprochen habe. Doch diese Konstruktion der Zusammenhänge kann es mit der rivalisierenden nicht an Wahrscheinlichkeit aufnehmen. Um nur eines hervorzuheben: Wir wissen nicht, bis zu welchem Grade Alkmaion sich pythagoreische Lehre zu eigen machte. Seine Tötung von Tieren zu wissenschaftlichen Zwecken ${ }^{15}$ läßt jedoch erkennen, daß er der Anschauung von der Seelenwanderung ablehnend gegenüberstand ${ }^{16}$. Wie hätte er sich sonst erkühnen können, Hand an Lebewesen zu legen, die nur zu leicht Inkorporationen der Seelen verstorbener Blutsverwandter darstellen konnten? So ist es fraglich, ob er mit den Pythagoreern in der Kugel das vollkommenste Gebilde des mathematischen Denkens erblickte.

In Medizinerkreisen vermochte Alkmaions grundlegende physiologische Einsicht in die Bedeutung des Gehirns sich nicht sofort durchzusetzen. In EMpedokles von Agrigent (5. Jahrhundert) erstand der Lehre des Krotoniaten ein gewichtiger Gegner, in dessen Person Philosophie und Heilkunst sich zu einem engen und fruchtbaren Bunde vermählten. Empedokles verkündete die Lehre, daß die geistige Tätigkeit genährt und unter-

\footnotetext{
13 Vorsokratiker $I^{6}, 212,21 \mathrm{ff}$. (Diels-Kranz).

14 Vorsokratiker $I^{6}, 212,16$ (DIELS-Kranz).

15 Vgl. Anmerkung ${ }^{11}$.

${ }^{16}$ Die Lehre von der Seelenwanderung ist eines der am sichersten für Pythagoras bezeugten Philosopheme; vgl. Vorsokratiker $I^{6}, 130,16 \mathrm{ff}$.
} 
halten werde von dem Blut, das das Herz umwallt ${ }^{17}$. Diese wissenschaftliche Ansicht hat weite Verbreitung gefunden und ein langes, zähes Leben geführt. Die Urgewalt des von dichterischer Begeisterung getragenen Wortes, die dem Arztphilosophen aus Agrigent zu Gebote stand und die über die Jahrhunderte hinweg den Römer Lucrez zu ehrfürchtiger Bewunderung hinri $\beta^{18}$, hat vermutlich das Ihre beigetragen zum siegreichen Vordringen der Anschauung vom Herzblut als Träger der Verstandestätigkeit, der gegenüber Alkmaions These zunächst einen recht schweren Stand hatte.

Die koische Schule scheint ihr jedoch Eingang gewährt zu haben. In der Schrift über die heilige Krankheit oder Fallsucht, in der man mit einiger Wahrscheinlichkeit ein Werk des Hippokrates selbst (zweite Hälfte des 5. Jahrhunderts) erkannt hat, wird dem Gehirn die bestimmende Rolle für das höhere Geistesleben zuerkannt und energisch die uralte Volksanschauung, die das Zwerchfell als Sitz des Denkens ausgab, zurückgewiesen ${ }^{19}$. Ebenso wird Empedokles' Annahme verworfen und sein Irrtum damit erklärt, daß starke Affekte wie auf die Trennungswand der oberen und unteren Leibeshöhle, so auch auf das Herz deutlich wahrnehmbare Auswirkungen haben. Die eigene Überzeugung hat sich dem Verfasser gebildet, weil er in Übereinstimmung mit einem zeitgenössischen Denker, Diogenes von Apollonia, im Element der Luft den Träger der Geistigkeit sieht und dieses auf seinem Weg in den Leib von der Nase ins Gehirn aufsteigen läßt. So scheinen hier Richtiges und Falsches sich zu einem merkwürdigen Bund zusammengefunden zu haben.

$\mathrm{Ob}$ auch in einer zweiten Schrift der hippokratischen Sammlung, die dem Ausgang des 5. Jahrhunderts vor Christus angehören mag und sich Über das Fleisch betitelt, Alkmaions Lehre ihren Niederschlag gefunden hat, ist zweifelhaft ${ }^{20}$. In unmißverständlichen Worten wird dem denkenden Geist sein Sitz nicht im Gehirn angewiesen; ob eine solche Annahme als stillschweigende Voraussetzung den Darlegungen zugrunde liegt, würde eine eingehendere Untersuchung verlangen.

Aristoteles (384-322) bietet seine gewaltige Autorität zugunsten der empedokleischen Lehrmeinung auf. Lebenswärme und Lebenskraft quellen für ihn aus dem Herzen. Hier beherbergt er die seelischen Prozesse, die nach seiner Meinung in enger Verbindung mit den Lebensvorgängen ste-

17 Vorsokratiker $I^{6}, 350,13$ (= 31 B 105) (Diels-Kranz).

${ }^{18}$ LUCREZ I, $716 \mathrm{ff}$.

${ }^{19}$ Corpus Hippocraticum, De morb. sacr. c. $16 \mathrm{f}=\mathrm{VI} 390 \mathrm{ff} \mathrm{L}$.

${ }^{20}$ Corpus Hippocraticum, De carn. c. $4=$ VIII $588 \mathrm{~L}$. 
hen ${ }^{21}$. Die zeitgenössischen und ein wenig jüngeren Ärzte haben sich der wissenschaftlichen Autorität des Stagiriten gebeugt ${ }^{22}$.

Wandel schafften die neuroanatomischen Forschungen der frühhellenistischen Ärzte Herophilos, Erasistratos und Eudemos. Hatte man bisher unter der Bezeichnung Nerv nicht nur die Nerven im heute geläufigen Sinn verstanden, sondern vor allem auch Sehnen und Flechsen, so wurde nun die Bezeichnung auf jene Organe begrenzt, welche vom Gehirn und Rückenmark ausgehen und die Leitungsbahnen für Empfindungen und Bewegungsimpulse sind. Indem man am lebenden Organismus Nervenleitungen durchtrennte, erkannte man, daß die Empfindungsfähigkeit gewisser Körperpartien und die Bewegungsfähigkeit bestimmter Muskeln verlorenging. In Medizinerkreisen gelangte damit Alkmaions Anschauung beinahe allgemein zu siegreichem Durchbruch ${ }^{23}$; nur die Pneumatiker (nachchristliche Zeit) versuchten für die verlorene Sache des Empedokles eine Lanze zu brechen, indem sie das von der Neuroanatomie gesammelte Beobachtungsmaterial durch sophistische Auslegungskünste in das Schema ihrer Theorie preßten ${ }^{24}$. Ganz zu schweigen von den führenden Philosophiesystemen des Hellenismus, Stoa und Epikureismus. In vornehmer Exklusivität gegenüber der Tatsachenforschung verharrend, nahmen sie keine Notiz von dem Fortschritt der Einzelwissenschaften und verteidigten den altüberkommenen Irrtum mit spitzfindigen Scheinargumenten.

Wo steht nun Plato in dieser Entwicklung? Was er im Timaios zu dieser Frage zu sagen hat, dürfen wir als einen Kompromiß zwischen den Antipoden Alkmaion und Empedokles ansehen. Im Staat hatte er seinen ursprünglichen Standpunkt von der Einheit der Seele aufgegeben und ihn durch die Auffassung ersetzt, daß die Seele ein mehrgliedriges Ganzes ist ${ }^{25}$. Diese Überzeugung hatte sich ihm aufgedrängt bei der Zergliederung des Seelenlebens, insbesondere jener Erscheinung, die man als Kampf der Motive bezeichnen kann. Die Beweggründe, die das menschliche Handeln bestimmen, sind, wenn man sittliche Maßstäbe anlegt, nicht alle von gleichem Wert. Plato glaubte drei Gruppen scheiden zu müssen und ordnete jeder Gruppe einen bestimmten Seelenteil zu: den Vernunftzwecken den

21665 a 12,$17 ; 666$ b $14 ; 456$ a 6 usw.

22 Diokles von Karystos: Anecd. Med. Fuchs, Rhein. Mus. 48, 543; Tertull. de anim.

c. 15. Praxagoras von Kos: Athen. $X V 687$ e.f. Kaibel.

23 Galenos VIII, $19 \mathrm{~K}$.

24 Galenos X, 929; VIII $19 \mathrm{~K}$.

${ }_{25}$ Politeia IV, 436 A ff. 
Geist, dem Verlangen nach Ehre und Ruhm den Mut, den niedrigen Trieben und Leidenschaften die Begierde. Mit seiner Psychologie verschmolz er nun die Lehren vom Seelensitz, wie Alkmaion und Empedokles sie entwickelt hatten. Er bestimmte dem unsterblichen und edelsten Seelenteil, dem Geist, seinen Sitz im Gehirn ${ }^{26}$. Mutmaßlich hat er Alkmaions Begründung gekannt; ob er sie billigte, kommt im Timaios nicht in klaren Worten zum Ausdruck. Hier steht eine andere Überlegung im Vordergrund, die vor der Vernunft als Wertbewußtsein die Kopplung von Hirn und Erkenntnisvermögen zu rechtfertigen sucht. Mit den Pythagoreern teilt Plato die Ansicht, daß unter den geometrischen Gebilden die Kugel als das vollkommenste angesprochen werden muß. Sie ist gestaltgewordenes Gleichund Ebenmaß und so im Bereich des Sinnlichen Verkörperung der Nämlichkeit, die, weil sie fest in ihrem Wesen ruht, als Höchststufe der Vollendung gelten kann. Der menschliche Schädel ist dem Inbegriff der Vollendung im Körperlich-Gestalthaften nachgebildet und deswegen am würdigsten, dem vornehmsten Seelenteil Quartier zu bieten, während der übrige Leib im Dienst der Aufgabe steht, das Haupt und seinen herrscherlichen Gast über die Unebenheiten des Erdbodens dahinzutragen. Den Menschen unserer Zeit dürfte es einige Mühe kosten, diese Gedanken nachzuvollziehen. Für Plato besitzen sie zwar keine zwingende Beweiskraft, aber doch viel unmittelbar Einleuchtendes.

Für die edlen Leidenschaften billigt er Empedokles' These eine beschränkte Geltung zu. Ihr Seelenorgan, den Mut, siedelt er im Herzen an ${ }^{27}$. Er geht dabei aus von Beobachtungen des Gefühlslebens, die bereits in der Abhandlung über die Fallsucht die Aufmerksamkeit des Verfassers erregt hatten. Zorn und Furcht äußern sich physiologisch in starkem Herzklopfen ${ }^{28}$. So liegt es ungemein nahe, den Träger der Ausdruckserscheinung mit dem der ausgedrückten Erscheinung in eins zu setzen. Die verhältnismäßige Einförmigkeit des physiologischen Korrelates im Vergleich zur bunten Vielfalt der Gefühlszustände, denen die Rolle auslösender seelischer Wirkkräfte zufällt, bleibt dabei außer Betracht, obwohl sie vor überhastiger Identifikation warnen könnte. Plato ist um so eher geneigt, solcherlei Bedenken hintanzustellen, als ihm die annähernd zentrale Lage des Herzens mit seiner leichten Verschiebung zu den oberen Körperpartien hin - der Linksverlagerung schenkt er keine Beachtung, wenn er sich ihrer überhaupt

${ }^{26}$ Timaios $44 \mathrm{D}$.

27 Timaios $69 \mathrm{C} \mathrm{ff.}$

${ }^{28}$ Timaios $70 \mathrm{C}$. 
bewußt war - ein höchst sinnvoller Baugedanke des göttlichen Menschenbildners zu sein scheint. Bereits im Staate hatte er mit der seelischen Einrichtung des Menschen die Schichtung des Gesellschaftskörpers parallelisiert. Dabei hatte dem mittleren Stand der Wächter im Staat der mittlere Seelenteil des Mutes im Einzelmenschen entsprochen. Fiel den Wächtern die Aufgabe zu, die Herrschaft der philosophischen Machthaber zu sichern und für die Einhaltung der von ihnen erlassenen Gesetze zu sorgen, so hatte der Mut darauf zu achten, daß die Gebote der Vernunft beim untersten Seelenteil williges Gehör fanden. Damit er seiner Kontrollaufgabe gewachsen ist, ist es zweckmä $\beta i g$, daß er seinen Sitz nicht allzu fern vom Gehirn und doch möglichst in der Leibesmitte hat ${ }^{29}$. So ist die Schnelligkeit der Befehlsübermittlung und leichter Überblick über den Kontrollbereich gewährleistet. Den niedrigeren Begierden schließlich wird die Leber zum Aufenthalt zugewiesen ${ }^{30}$. So baute Plato eine alte Volksmeinung in sein System ein. Von seiten der Mediziner hat diese Meinung keine Unterstützung erfahren. Doch hat sie sich im Bereich volkstümlicher Vorstellungsweise lange halten können und spielt selbst heute noch eine gewisse Rolle.

Die Lehre Platos von der Vereinigung von Körper und Seele bietet uns ein recht instruktives Beispiel dafür, wie der Philosoph, durchdrungen von dem Glauben, daß der Geist der Ordnung und Vernunft die empirische Wirklichkeit durchwaltet, widerstreitende Strömungen in der Naturwissenschaft seiner Tage zum Ausgleich zu bringen sucht. Der Philosophie als Königin unter den Wissenschaften, die sich im 4. Jahrhundert langsam aus ihrem Schoß zu lösen beginnen, wofern sie nicht wie die Heilkunde von jeher Eigenständigkeit besaßen, wird somit das ehrenvolle Amt übertragen, als Schiedsrichter im Streit der Meinungen in letzter und höchster Instanz die Entscheidung zu fällen.

\section{d) Atmung}

Seele, Leben und Atmung bilden für den antiken Menschen eine enge und oft sogar unauflösliche Einheit. So ist es begreiflich, daß Plato das Einziehen und Ausstoßen von Luft mit angelegentlichem Interesse studierte und dem Gegenstand eine ausführliche Darstellung widmete ${ }^{31}$. Schon vor ihm hatte sich die Naturbetrachtung diesem Problem zugewandt und sein Geheimnis zu ergründen versucht. Wie schwierig es war, richtigen Anschau-

29 Timaios 70 A ff.

30 Timaios $70 \mathrm{D}$ ff.

31 Timaios $77 \mathrm{C} \mathrm{ff.}$ 
ungen Bahn zu brechen, mag die Tatsache beleuchten, daß erst der athenische Staatsmann und Verfassungsreformer Solon (Erste Hälfte des 6. Jahrhunderts) die für uns selbstverständliche Wahrheit ausspricht, daß die Lunge ein Atmungsorgan ist und nicht ein Aufnahmebehälter für flüssige Nahrung, ein Irrtum, der sich noch viele Jahrhunderte hat behaupten können, wenn auch nicht in der ursprünglichen rohen Form. Einer der ersten, die sich über den Atmungsprozeß Klarheit zu verschaffen suchten, ist Empedokles ${ }^{32}$. Ein langes Bruchstück aus seinem Lehrgedicht ist auf uns gekommen, in dem er seine Anschauung über den Gegenstand weitläufig auseinandersetzt. Der Atmung dienen nicht nur die Nasenlöcher, sondern gleichfalls kleine Öffnungen, mit denen die Hautoberfläche des Leibes übersät ist. Ihre Kleinheit gestattet dem Blut den Austritt nicht, wohl aber der Luft den Eintritt. Empedokles sieht eine enge Verbindung zwischen der Blutbewegung und dem Respirationsprozeß. Das Blut befindet sich in ständigem Anschwellen und Absinken, vergleichbar dem Steigen und Fallen des Meeresspiegels bei Ebbe und Flut. Strömt es im Körperinnern zusammen, so dringt die Außenluft durch die Nase und die Hautporen ins Körperinnere; strömt es in Richtung auf die Körperoberfläche zurück, wird die Luft auf dem gleichen Wege hinausgepreßt. Durch einen Vergleich mit dem Wasserheber, der sich dem modernen Verständnis nur schwer erschließt, ist er bestrebt, seine physiologische Anschauung zu verdeutlichen. Für die Auf- und Abwärtsbewegung des Blutes scheint er keine Erklärung gegeben zu haben. Der Ausdruck, den Aristoteles zur Wiedergabe dieser Lehre benutzt, deutet darauf hin, daß der agrigentinische Denker sie aus dem Wesen des Blutes ableitete, das heißt, sie als eine gegebene Tatsache hinnahm und keine Anstalten traf, sie auf tieferliegende, allgemeinere Gesetzmäßigkeiten zurückzuführen ${ }^{33}$. Auch ist nicht kenntlich, welche Vorstellungen ihn veranlaßt haben mögen, in dem rhythmischen Auf und Ab ein Wesensmerkmal des Körpersaftes zu erblicken.

Plato baut seine Theorie der Atmung unter starker Verwendung Empedokleischer Elemente auf. Doch hat er das verhältnismäßig einfache Schema des sizilischen Arztes abgewandelt und weiter ausgebaut. Auch er nimmt eine Nasenatmung und eine Hautatmung an ${ }^{34}$. Ein- und Ausatmung erfolgt jedoch nicht mehr auf beiden Wegen gleichzeitig, sondern im Wechsel. Der Einatmung durch die Nase entspricht bei Plato Ausatmung durch die

${ }^{32}$ Vorsokratiker $I^{6}, 347,13 \mathrm{ff} .=31$ B 100 (Diels-Kranz).

${ }^{33}$ Aristoteles, De respir. 473 b 5.

${ }^{34}$ Timaios $79 \mathrm{~A} \mathrm{ff}$. 
Haut und umgekehrt. Diese einschneidende Änderung rührt daher, daß bei dem athenischen Philosophen die Luftbewegung nicht mehr von dem Auf- und Abwogen des Blutes im Körperinnern abhängig gemacht wird, sondern mit der eingepflanzten Wärme in Beziehung gesetzt ist. Diese Kraft, die für die Unterhaltung des Lebensprozesses von großer Bedeutung ist, verteilt sich im gesamten Körperinnern, hat jedoch ihr eigentliches Wirkzentrum im Blut und in den Adern ${ }^{35}$. Es hat nicht an Bemühungen gefehlt, auch für Plato wie für Aristoteles das Herz als Herd der Lebenswärme in Anspruch zu nehmen; bei der Unzulänglichkeit der Beweismittel ist es kaum erstaunlich, daß diesen Versuchen der Erfolg versagt blieb. Um den Verlauf des Atmungsprozesses im einzelnen zu erklären, werden zwei Prinzipien herangezogen: Erstens leugnet Plato das Vorhandensein des leeren Raumes ${ }^{36}$. Bewegung einer Stoffpartikel bedeutet darum immer Verdrängung anderer materieller Teile von ihrem Platz. Das Beispiel eines schwimmenden Fisches, vor dem sich beim Durchqueren das Wasser zerteilt und hinter dem es sich wieder zusammenschließt, mag zur Veranschaulichung dienen. Zweitens geht Plato von dem Grundsatz aus, daß Gleiches zu Gleichem strebt ${ }^{37}$. So suchen sich die feurigen Partikel im Inneren des Leibes mit dem feurigen Stoff außer uns zu vereinigen. Die durch die Hauptporen eingetretene Luft wird im Körperinnern erwärmt und strebt nach ihrer Erwärmung auf demselben Weg zur Außenluft zurück. Da es kein Leeres gibt, treibt sie, ohne daß ein zeitliches Intervall einträte (wie bei der Drehung eines Rades) ${ }^{38}$, die kühle Außenluft auf dem Nasenweg in die Leibeshöhle. Die auf diesem Weg eingedrungene Luft unterliegt nun ihrerseits der Erwärmung durch das eingepflanzte Feuer und hat dementsprechend das Bestreben, auf demselben Weg den Körper zu verlassen und preßt dabei kühle Außenluft durch die Haut in den Leib. So beginnt das Spiel von neuem. Man braucht dieses Gedankengewebe nicht allzu kritisch unter die Lupe zu nehmen, um zu bemerken, daß es mit einer Anzahl von Schwächen und Unzulänglichkeiten behaftet ist. So hat die Theorie gleich den Schüler Aristoteles zu lebhaftem Widerspruch herausgefordert ${ }^{39}$. Aristoteles tadelt mit Recht, daß Platos Betrachtungsweise im Äußerlichen steckenbleibt und keinen Aufschluß über den Zweck des ständigen Wechsels von

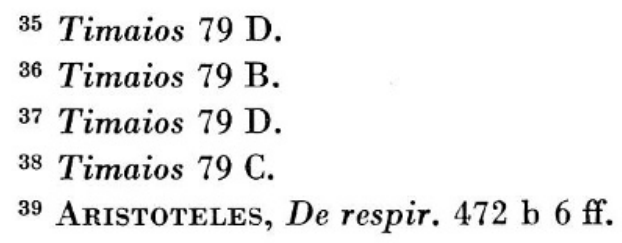


Ein- und Ausatmung gibt. Neuere Erklärer haben diesem Einwand zu begegnen versucht und dargelegt, daß durch die ein- und ausströmende Luft das innere Feuer in oszillierende Bewegung gesetzt wird und auf diese Weise das Blut, mit Nahrungsstoffen angereichert, an alle Organteile des Körpers gepumpt wird. Die Bemerkung ist so unrichtig nicht ${ }^{40}$, wird aber von Plato nicht als das Ziel hingestellt, dem die Entwicklung der Gedanken zustrebt.

Gleichfalls legt GaLen (etwa 129 bis etwa 199 nach Christus) den Finger auf eine wunde Stelle. Für Plato ist der Vorgang des Atmens ein rein mechanischer Prozeß, auf den der Mensch so wenig wie auf die Herzbewegung Einfluß nehmen kann. Und doch lehrt die alltägliche Erfahrung, daß wir die Atemzüge beschleunigen und verlangsamen, den Atem für eine gewisse Zeit anhalten, wenn auch nicht ganz bis zum Erstickungstode unterdrücken können, kurzum, dem bewußten Willen eine gewisse Steuerungsmöglichkeit über die Respiration eingeräumt ist ${ }^{40^{\mathrm{a}}}$.

Trotz ihrer offen zutage liegenden Unzulänglichkeiten kommt der Platonischen Atmungstheorie das Verdienst zu, energisch das Interesse der wissenschaftlichen Welt auf den Atmungsvorgang gelenkt zu haben. Seitdem haben Philosophie und Naturwissenschaft sich in hingebungsvoller Arbeit in den Gegenstand vertieft, und es ist der antiken Forschung vergönnt gewesen, ein tüchtiges Stück über Plato hinaus in Richtung auf die Wahrheit vorzudringen. Ferner war es ein fruchtbarer Gedanke, das angeborene Lebensfeuer mit der Luftaufnahme und -abgabe in Beziehung zu setzen. Hatte Plato den hier obwaltenden Zusammenhang mechanisch-kinetisch aufgefaßt, so bildete dies für den Schüler Aristoteles einen Ansporn, im edlen Wettstreit mit dem Lehrer dessen Auffassung durch eine berichtigte und vertiefte Betrachtungsweise zu ersetzen. Er stellte sich die Rolle, welche die Luft gegenüber der Lebenswärme spielt, nach Analogie ihrer Mitwirkung beim Feuer, bei der Verbrennung vor. Wie die Flamme beim Abschneiden der Luftzufuhr zum Erlöschen verurteilt ist, so kann die Lebenswärme einem Erkalten im Tode nicht entgehen bei Unterbindung der Atmung ${ }^{41}$. Bei der Deutung des Phänomens der aus Luftmangel ersterbenden Flamme geriet er allerdings auf Abwege. Bei Aristogenes, dem Leibarzt des mazedonischen Königs Antigonos Gonatas (erste Hälfte des dritten Jahrhunderts), stoßen wir dann auf die bedeutsame Ansicht, daß die Luft Nahrung ist,

40 Timaios $78 \mathrm{E} \mathrm{ff}$.

40a Galenos $V 712 \mathrm{~K}$

${ }^{41}$ Vgl. Aristoteles, De respir. 474 a $25 \mathrm{ff}$. 
d.h. für die stoffliche Ergänzung der organischen Substanz unentbehrlich, und in der Lunge eine Art Verdauungsprozeß durchläuft wie die Speise im Magen ${ }^{42}$. Wenn wir schließlich bei Galen lesen, daß bei der Ausatmung rußartige Stoffe ausgeschieden werden, so sind wir durch die (scheinbare) Modernität einer solchen Lehre überrascht. Doch blieb es der antiken Wissenschaft versagt, den Schleier vom Geheimnis der Atmung zu heben. Das liegt vor allem an dem Fehlen einer chemischen Wissenschaft, die ja bekanntlich erst in der Neuzeit durch den Engländer BoyLE ins Leben gerufen wurde, und erst das Zeitalter der Aufklärung vermochte es, nachdem die Luft als ein Gemisch aus Stickstoff, Sauerstoff und Kohlenstoff erkannt war, das Rätsel der Atmung zu lösen und ihr Wesen als Gasaustausch zu bestimmen. Die Namen des englischen Arztes John Mayow (17. Jahrhundert) und vor allem von Priestley und Scheele (18. Jahrhundert), die ungefähr gleichzeitig und unabhängig von einander den Sauerstoff entdeckten, bezeichnen die hervorragendsten Meilensteine auf diesem mühevollen Weg, an dessen Ziel die glänzende Gestalt Lavoisiers steht.

Gleichberechtigt neben der Frage nach der historischen Bedeutung steht die nach den geistigen Ursprüngen der Platonischen Atmungstheorie. Denn die aufgezeigten Abweichungen von Empedokles verlangen gebieterisch nach einer Erklärung. Hier tappen wir einigermaßen im dunkel. Vielfach wird Philistion, ein Zeitgenosse Platos, der am Hofe des Dionysios von Syrakus die Stelle eines Leibarztes bekleidete, als der Mittelsmann ausgegeben, der Empedokleisches Gedankengut mit seinen eigenen Anschauungen und Erkenntnissen bereicherte und so an Plato weitergab. $\mathrm{Daß}$ der syrakusanische Hofarzt neben der Nasen- auch die Hautatmung annahm, ist gut verbürgt und unterliegt kaum einem Zweifel ${ }^{43}$. Daneben soll er nach einer nicht durchaus verläßlichen Quelle die Kühlung des eingepflanzten Feuers als Zweck der Atmung bezeichnet haben ${ }^{44}$. Die Richtigkeit dieser Nachricht vorausgesetzt, öffnet sich hier zwischen ihm und dem athenischen Weisen eine trennende Kluft. Nach Plato nämlich erstreckt sich die kühlende Wirkung der Atemluft auf alle Teile des Leibes, nicht nur auf das Blut, in dem er den Sitz der Lebensglut in Übereinstimmung mit Empedokles vermutet ${ }^{45} . \mathrm{Ob}$ es der historischen Forschung je gelingen wird, Licht über diesen dunklen Punkt zu verbreiten, läßt sich mit Sicherheit nicht voraussagen.

42 Pseudo-Aristoteles 481 a $28 \mathrm{ff}$.

43 Philistion, frg. 4 (Wellmann).

${ }^{44}$ Galenos $I V 471 \mathrm{~K}=$ frg. 6 (Wellimann).

45 Timaios $84 \mathrm{D}$. 


\section{e) Verdauung}

Spielte die eingepflanzte Wärme eine hervorragende Rolle in der Platonischen Respirationstheorie, so hat sie in der Verdauungstheorie des Philosophen einen Platz von mindestens gleicher Bedeutung inne. Mit Platon hebt das Studium der Physiologie der Nahrungswandlung in körpereigene Substanz keineswegs an. Die Lösungsversuche dieser Frage können zu seiner Zeit schon auf eine längere Vorgeschichte zurückblicken. Einer der frühesten, die sich mit einer Theorie hervorwagen, ist wiederum Empedokles. Freilich haben sich von seiner Lehre nur kärgliche Spuren erhalten ${ }^{46}$. Er erblickte in der Nahrungsumwandlung einen Fäulnisvorgang. PleistoNeIKos, wie der oben erwähnte Herophilos ein Schüler des Praxagoras von Kos (Ausgang des 4. Jahrhunderts vor Christus), hat diese Vorstellung aufgegriffen und bei einer Anzahl von Ärzten damit Anklang gefunden ${ }^{47}$. Wie man sich den Vorgang im einzelnen vorstellte, ist. uns wegen der Spärlichkeit der Nachrichten nicht mehr kenntlich. In der modernen historischen Forschung wird gelegentlich die Ansicht verfochten, daß bei der Fäulnis- oder Fermentationstheorie der Wirkzusammenhang zwischen dem eingepflanzten Feuer und der Verdauung aufgehoben ist. Eine solche Annahme zum Rang einer gesicherten Erkenntnis zu erheben, dürfte kaum im Bereich des Möglichen liegen.

Plato zeigt größere Verwandtschaft zu den Vorstellungen, die sich in einigen Schriften der Hippokratischen Sammlung finden. Hier wird vor allem die Analogie hervorgehoben, in welcher der Verdauungsvorgang zum Kochen steht. Rohe Nahrung ist dem Leib im allgemeinen nicht zuträglich. Durch Einwirkung des eingepflanzten Feuers müssen die Speisen in den Zustand des Garseins übergeführt werden, sollen sie die Einbuße des Leibes an organischen Stoffen der verschiedensten Art wettmachen.

Diese Gedanken finden in Platos Timaios ihre Ausgestaltung. Nahrung nimmt der Mensch in fester und flüssiger Form zu sich, veranlaßt durch die Nahrungstriebe, Hunger und Durst, die ihren Sitz, wie bereits bemerkt, in der Leber haben. Weise ist es von den Göttern eingerichtet, eine so große Entfernung zwischen die Residenz des Geistes und der Vernunft, das Haupt, und den dritten und niedrigsten Seelenteil zu legen. So vermag seine Unruhe ich nicht störend auf das Geistesleben auszuwirken ${ }^{48}$.

S

4

${ }^{4}$ Vorsokratiker $I^{6}, 299,2$ (DiEls-Kranz).

${ }^{4}$ Celsus I prooem $20 \mathrm{ff}$. (MARx).

${ }^{4}$ Timaios $70 \mathrm{E}$. 
Die feste Nahrung gelangt samt und sonders in den Magen, die flüssige zum größten Teil; doch wird eine gewisse Menge durch die Lunge aufgenommen und von hier zu den Nieren und zu der Blase weitergeleitet ${ }^{49}$. Der Philosoph hat sich nicht im einzelnen darüber ausgesprochen, wie man sich die Verbindung zwischen der Lunge und den Nieren zu denken hat. Überhaupt liegt über seinen Ausführungen, die sich mit der Flüssigkeitsaufnahme befassen, eine gewisse Unklarheit, so daß sich in der antiken Timaios-Erklärung die Meinung hat bilden können, daß der Weg für die gesamte flüssige Nahrung durch die Lunge führe. Schon Aristoteles ist dem Irrtum, daß die Luftröhre als Durchgang für die Flüssigkeit diene, nachdrücklich entgegengetreten ${ }^{50}$. Er erwies sich aber als unausrottbar, selbst nachdem die Koryphäe der hellenistischen Medizin, Erasistratos, über Platos anatomischer Ignoranz die Schale ihres Spottes ausgegossen hatte ${ }^{51}$.

Doch lassen wir die Fragen, die mit dem Weg der Nahrungsaufnahme zusammenhängen; richten wir den Blick auf das eigentliche Verdauungsproblem! Die dem Magen und Leib zugeführten Speisen unterliegen der Einwirkung des inneren Feuers. Im Gleichklang mit den Luftmassen, die durch die Hautoberfläche und die Nasenöffnungen ein- und ausströmen, befindet sich dieses im Zustand des Hin- und Herschwingens. Die Elementenlehre hatte dargelegt, daß sich der Grundstoff des Feuers aus Pyramiden aufbaut, eine Struktur, die nicht nur einen ausreichenden Erklärungsgrund für seine Leichtigkeit und Beweglichkeit, sondern auch für seine Schärfe bietet. Dank ihrer Scharfkantigkeit sind die feurigen Stoffteilchen in der Lage, die in den Magen hinabgleitende Nahrung zu zerschneiden und in einen flüssigen Nahrungssaft, das Blut, zu verwandeln, das, weil es stark mit Feuerpartikeln angereichert ist, rote Färbung zeigt. Gleichzeitig wird durch das Hin- und Herpendeln des inneren Feuers das Blut aus dem Verdauungsorgan in die Blutgefäße gepumpt und verteilt sich von hier aus über den ganzen Körper und sämtliche Organe ${ }^{52}$.

Obwohl Plato das Herz Knotenpunkt der Adern und Quelle des im Körper umlaufenden Blutes nennt ${ }^{53}$, ist er doch weit davon entfernt, zu ahnen, daß die Blutbewegung durch die Wirksamkeit des Herzens verursacht ist und in einer geschlossenen Bahn verläuft, so daß man von einem Kreislauf

\footnotetext{
49 Timaios 91 A.

50 Aristoteles 664 b 6 ff.

${ }^{51}$ Macrob. 7, 15, $1 \mathrm{ff}$.

52 Timaios 78 E f.

53 Timaios $70 \mathrm{~B}$.
} 
zu sprechen berechtigt ist. Der hellenistische Physiologe Erasistratos stand auf Grund seiner intimen Kenntnis des Klappenapparates des Herzens dicht vor dieser Einsicht, doch war er zu tief in der von Praxagoras von Kos in die Welt gesetzten Irrlehre verstrickt, daß die Arterien nur Luft und kein Blut führten, um den letzten entscheidenden Schritt zu tun und zur Wahrheit durchzustoßen. So blieb es HARveY vorbehalten, nach zahllosen Experimenten den wahren Sachverhalt ans Tageslicht zu bringen und so die Physiologie auf eine völlig neue Grundlage zu stellen.

Plato unterscheidet auch noch nicht zwischen Arterien und Venen. Für ihn wie für Aristoteles gibt es nur eine einzige Klasse von Blutgefäßen. Die Medizin verdankt die Trennung von Schlag- und Blutadern Praxagoras, der mit ihr allerdings einen anderen Sinn verband, als wir es heute zu tun pflegen ${ }^{54}$. Denn bei der Statuierung des Unterschiedes ging er nicht von der Blutbewegung aus, sondern von der Erscheinung des Pulsierens, deren Kenntnis zwar schon bis in die Hippokratische Sammlung zurückreicht, die aber noch ein Aristoteles nicht auf die Schlagadern allein zu begrenzen gelernt hatte ${ }^{55}$.

Doch kehren wir zum Blut zurück. Nachdem es durch das Blutgefäßsystem an sämtliche Körperstellen herangetragen worden ist, saugen die einzelnen Organe die Bestandteile aus ihm, die ihnen gemäß sind. Denn sie bedürfen einer Erneuerung des Stoffes, aus dem sie aufgebaut sind, da sie unter der Einwirkung von Umwelteinflüssen ständig Substanzverluste erleiden.

In der Platonischen Verdauungstheorie sind sehr verschiedenartige Elemente zu einer Einheit verschmolzen. Beschränkung auf das Wichtigste sei gestattet. Wie flüchtig berührt, dürfte die allgemeine Analogie zum Prozeß des Kochens der hippokratischen Medizin entlehnt sein. Bei der Zerschneidung des Nahrungsbreis durch die scharfkantigen Feuerpartikel hat der Atomismus Demoкrits (5. Jahrhundert) Pate gestanden. Plato ist bei dem Abderiten in die Schule gegangen und hat von ihm den Gedanken übernommen, daß kleinste Teilchen die Materie aufbauen. In einem bedeutsamen Punkt freilich schlägt er seine eigenen Wege ein. Während Demokrit eine unbeschränkte Gestaltenfülle der Atome annahm, läßt Plato nur vier Grundformen für die letzten Bausteine der Materie gelten, entsprechend der Vierzahl der empedokleischen Elemente, und wählt sie unter

54 Galenos VIII 941; 950; 702; $498 \mathrm{~K}$.

55 Aristoteles, 480 a 11. 
den sogenannten platonischen Körpern aus. Mit der Mathematisierung der letzten unzerlegbaren Stoffeinheiten ist der Rationalität Eingang in den Bereich des Materiellen verschafft.

Platos Theorie ist keineswegs das letzte Wort der antiken Naturwissenschaft zum Verdauungsproblem. Die nachfolgenden Jahrhunderte haben noch viele bemerkenswerte Gedanken zutage gefördert. Auch hier geht von der physiologischen Erkenntnisarbeit des Erasistratos ein gewaltiger Impuls aus. Der Keer warf die Lehre vom eingepflanzten Feuer über Bord ${ }^{56}$ und bemühte sich an die Stelle der thermodynamischen Verdauungslehre eine rein mechanische Erklärung zu setzen ${ }^{57}$. Man war auf die Peristaltik des Magens und Darms aufmerksam geworden. Daraus entwickelte sich eine doppelte Auffassung von der Leistung der Verdauungsorgane: Sie hatten zunächst die Aufgabe, die Nahrung zu zerreiben und hernach den so entstandenen Saft in die Blutadern zu pressen ${ }^{58}$.

Wie verschieden ist hiervon die Rolle, die der Darm bei Plato spielt! Er dient ausschließlich dem Abtransport der Abfallprodukte der Verdauung. Die Götter haben ihn lang und windungsreich gebildet, um das Menschengeschlecht vor vorzeitigem Leeregefühl und damit dem Laster der Gefräßigkeit zu bewahren ${ }^{59}$. Frei von der Fessel eines unstillbaren Nahrungstriebes und begabt mit dem sonnenhaften Organ des Auges ${ }^{60}$ soll der Mensch im Aufblick zum Sternenhimmel mit seiner strengen Ordnung und majestätischen Pracht sich der Wahrheitserkenntnis weihen und seines göttlichen Ursprungs sowie seiner göttlichen Bestimmung innewerden. Dies ist ein besonders eindringliches Beispiel dafür, wie innig sich bei Plato Ethik und Naturbetrachtung durchdringen.

\section{f) Krankheit und Tod}

An die Betrachtung der Lebensvorgänge im Zustand der Gesundheit fügt Plato eine allgemeine Krankheitslehre ${ }^{61}$. Er gruppiert die Gesundheitsstörungen in drei große Klassen. In der ersten Gruppe faßt er die Krankheiten zusammen, die in einem Mißverhältnis der Elementenmischung wur-

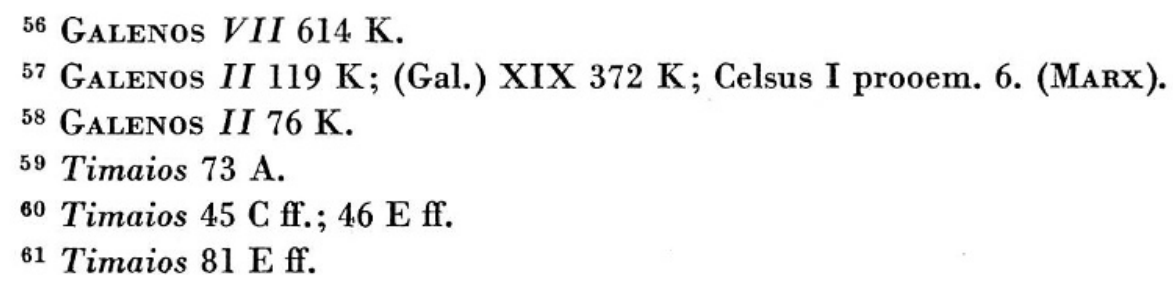


zeln $^{62}$. Die zweite Gruppe wird durch diejenigen Leiden gebildet, bei denen die Vorgänge der Substanzerneuerung durch die Ernährung in Unordnung geraten sind ${ }^{63}$. In der dritten finden sich all die pathologischen $\mathrm{Zu}$ stände vereinigt, deren Ursache entweder in der anomalen Versorgung mit Atemluft oder in dem schädlichen Einfluß von Galle oder Schleim zu suchen ist ${ }^{64}$.

Ergänzen wir diesen allgemeinen Überblick durch einige Einzelbemerkungen, ohne uns zu sehr ins Detail zu verlieren! Der Leib des Einzelwesens baut sich wie die umgebende Welt aus den vier Elementen auf, die sich, um die organischen Substanzen, wie Mark, Knochen, Fleisch usw., hervorzubringen, in bestimmten Mischungsverhältnissen miteinander verbinden. Die eigentliche Ursubstanz des Organismus ist das Mark, zu dessen Bildung die vier Elemente mit besonders rein und regelmäßig geformten Urdreiecken verwendet wurden. Die Knochen wurden aus reiner, glatter, mit Mark zusammengekneteter Erde geformt und durch mehrmaliges Eintauchen in Wasser und Feuer gehärtet. In ähnlicher Weise kamen die übrigen Organsubstanzen zustande. Wird das ursprüngliche Mischungsverhältnis in ihnen aus dem Gleichgewicht gebracht, dann wird der Leib von Krankheiten befallen. Die Organe verändern die ihnen von Natur zukommende Beschaffenheit, sind überhitzt oder unterkühlt, oder der ihnen gemäße Feuchtigkeitsgehalt erfährt Veränderungen im Sinne eines Zuviels oder Zuwenigs.

In dieser Anschauung sind zwei Betrachtungsweisen miteinander aufs innigste vermählt. Der Gedanke, daß Gesundheit auf Harmonie und Ausgewogenheit der Kräfte des Leibes beruht, war schon seit alten Zeiten im ärztlichen Denken der Griechen heimisch. Besonders prägnanten Ausdruck hat ihm Alkmaion in einem berühmten Bruchstück verliehen ${ }^{65}$. Dieser Grundgedanke ist im Sinn der empedokleischen Vierelementen- und Vierqualitätenlehre ausgestaltet worden. Nicht Plato war es, der diesen Schritt getan hat. Er läßt sich schon für Philistion von Lokroi nachweisen ${ }^{66}$.

Gehen wir zur zweiten Klasse von Krankheiten über! Es wurde bereits erwähnt, daß sämtliche organische Gewebe zu ihrer Erhaltung des Blutes bedürfen, dessen Flüssigkeit die zum Ausgleich eingetretener Verluste be-

${ }^{62}$ Timaios $81 \mathrm{E}$ ff.

${ }^{63}$ Timaios $82 \mathrm{~B}$ ff.

${ }^{64}$ Timaios $84 \mathrm{Cff}$.

65 Vorsokratiker $I^{6}, 215,11 \mathrm{f}$. $=24$ B 4 (DIELs-Kranz).

${ }^{66}$ Anon. Londin.XX $25 \mathrm{ff}$. (Diels). 
nötigten Stoffe enthält. Nun kann es vorkommen, daß die Gewebe, anstatt aus den Nahrungsstoffen des Blutes ihre Substanzverluste zu ersetzen, zerfallen und die Zerfallsprodukte in das Blut ausscheiden. Am stärksten ist das Fleisch dieser Gefahr ausgesetzt. Auf diese Weise tritt eine Zersetzung des Blutes ein, bei der sich die verschiedenen Arten von Galle und Schleim bilden, die, vom Blutstrom umhergetragen, im Organismus großes Unheil anrichten können. Die Theorie erinnert von fern an humoralpathologische Auffassungen der Hippokratischen Sammlung, unterscheidet sich aber von ihnen in charakteristischer Weise dadurch, daß den Säften als solchen eine gesundheitsschädigende Wirkung zugesprochen wird und nicht ihrem verkehrten Mischungsverhältnis. Es ist bis auf den heutigen Tag nicht gelungen, diese Platonische Lehre in die Überlieferungsströme der griechischen Medizin einzuordnen ${ }^{67}$.

Festeren Boden fühlen wir unter den Füßen, wenn in der dritten Klasse von Gesundheitsstörungen die Verschleimung der Lunge und die dadurch bedingte mangelhafte oder überreichliche Belüftung der verschiedenen Körperteile als Krankheitsursache aufgeführt wird. Hier lehnt sich Plato an Philistion an, für den gleichfalls die Hemmung der Belüftung des Leibes in der Ätiologie der Krankheiten eine hervorragende Rolle spielt ${ }^{68}$. Der Rest der Krankheitslehre hingegen klingt in manchem an hippokratische Vorstellungen an, ohne daß sich genau bestimmen ließe, welchen Quellen er verpflichtet ist.

Daß von der Krankheitslehre vielfältige Verbindungsfäden zur Physiologie des Todes hinüberlaufen, ist nur natürlich. Im Lichte seiner Seelenanschauung war Plato schon früh der Tod als eine Trennung von Leib und Seele erschienen ${ }^{69}$. Mit der Geburt senkt sich die Seele in ihre irdische Hülle ein, herabsteigend aus den Lichtgefilden des überhimmlischen Ortes, wo sie dem Zuge der unsterblichen Götter folgend in seliger Ideenschau verweilt hatte ${ }^{70}$; mit dem Tod kehrt sie, wenn sie sich während ihres Erdenwandels unbefleckt von Schande und rein von Schuld bewahrt hat, in ihre überirdische Heimat zurück. Der Timaios ergänzt die metaphysischen Vorstellungen nach der physiologischen Seite hin. Wir hörten bereits von der Ansiedlung der drei Seelenteile in den verschiedenen Körperregionen. Das eigentliche Band, welches die Seele an den Leib kettet, ist das Gehirn und seine

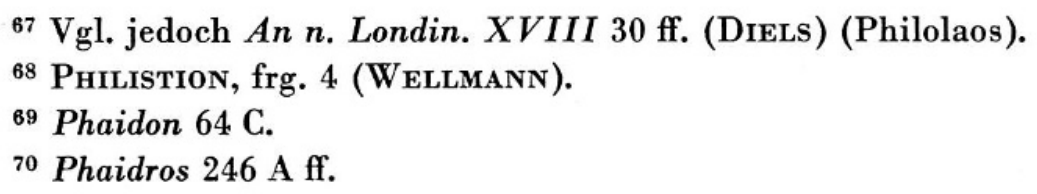


Fortsetzung, das Rückenmark. Von hier verlaufen Halteseile zu den Organen der sterblichen Seelenteile, Herz und Leber ${ }^{71}$. Über dem anatomischen Substrat dieser Stricke liegt dichtes Dunkel. Löst sich die Verkettung der Seelenteile mit dem Hirn und seiner Verlängerung, dann entweicht der unsterbliche Teil, während Mut und Begierde mit dem Leibe dem Untergang und der Vernichtung verfallen.

Zwei Ursachen für das Reißen der Seelenbande stehen einander in scharfer Scheidung gegenüber: eine natürliche und eine gewaltsame. Der natürliche Tod tritt ein, wenn im Verlauf des Alterns die ursprünglich scharfen Dreiecke, nachdem sie viele Jahre hindurch die Nahrungsteilchen aufgespalten haben, stumpf geworden sind und damit ihre Fähigkeit, die Nährstoffe zu zerteilen, mehr und mehr einbüßen. Die Folge ist, daß der Substanzverlust nur unvollkommen ergänzt werden kann und schließlich gar die Nahrungspartikel dank ihrer größeren Spitzigkeit die Elementarteilchen des Organismus auseinandertreiben. Ein Gefühl der Freude begleitet diese natürliche Auflösung ${ }^{72}$.

Schmerzhaft dagegen ist der gewaltsame Tod, der sich infolge von Verwundung und Krankheit einstellt ${ }^{73}$. Den Tod infolge Verwundung würdigt Plato keiner ins einzelne gehenden Behandlung. Auch der körperliche Untergang infolge jener Krankheiten, die auf regelwidriger Elementenmischung beruhen, wird nur flüchtig gestreift ${ }^{74}$. Bei den übrigen Krankheiten wird jedoch nachdrücklich betont, daß sie unweigerlich zum Tod führen, wenn die eigentliche Lebenssubstanz, Hirn und Mark, um die sich der übrige Körper wie ein schützender Panzer legt, in Mitleidenschaft gezogen wird ${ }^{75}$. In der vorsokratischen Naturphilosophie und Medizin war bereits eine große Mannigfaltigkeit von Hypothesen über die Ursache des Todes aufgestellt worden. Empedokles sah eine enge Verwandtschaft zwischen Schlaf und Tod und vermutete einen Zusammenhang zwischen den eingeborenen, im Blut beheimateten Wärme mit den beiden Erscheinungen. Bei teilweiser Abkühlung der Lebensglut umfing den Menschen der Schlaf, bei gänzlicher der Tod ${ }^{76}$. Aus dem Blut versuchte auch Alkmaion das Erklärungsprinzip für das zeitweise und endgültige Erlöschen des Be-

71 Timaios 73 B.

72 Timaios $81 \mathrm{C} \mathrm{ff.}$

73 Timaios $81 \mathrm{E}$.

${ }^{74}$ Timaios $82 \mathrm{~B}$.

75 Timaios $84 \mathrm{C} ; 85$ E.

${ }^{76}$ Vorsokratiker $I^{6}, 301,17 \mathrm{ff}$. (Diels-Kranz). 
wußtseins zu gewinnen ${ }^{77}$. Sammelt es sich vorübergehend in den sogenannten blutführenden Gefäßen, die sich vielleicht mit den Venen der modernen Anatomie decken, versinkt der Mensch in Schlaf; der Tod tritt ein, wenn überhaupt kein Rückfluten des Blutes in die Schlagadern mehr erfolgt. Platos Arztfreund Philistion führte den Tod vielleicht auf das vollständige Erliegen der Haut- und Körperatmung zurück ${ }^{78}$. Noch manche Ansicht ließe sich anführen. Aber soweit wir auch den Blick umherschweifen lassen, wir vermissen Gedankenkonstruktionen, die auf Grund ihrer schlagenden Ähnlichkeit uns ermutigen könnten, sie als Platonisches Vorbild anzusprechen. Schule hat Plato mit seiner Physiologie des Todes keine gemacht. Sie war mutmaßlich zu sehr mit den Elementen seiner Seelenmetaphysik und seiner mathematischen Auffassung vom Aufbau der Materie versetzt. Der Schüler Aristoteles griff über den Lehrer hinweg auf die altüberkommene Empedokleische Ansicht zurück und erblickte im Tod ein Erlöschen der Lebenswärme, die er sich allerdings nicht mehr an das Blut, sondern an das Herz, und zwar vornehmlich an die rechte Herzkammer, gebunden dachte. Die hellenistischen Ärzte scheinen, wenn man auf Grund des trümmerhaften Zustandes der Überlieferung einen solchen Schluß wagen darf, auf die Aufstellung einer allumgreifenden Todestheorie verzichtet zu haben. Galen schließlich empfindet starke, schier unüberwindlich dünkende Schwierigkeiten, wie er die Platonische Seelen- und Todeslehre mit gewissen unbestreitbaren Gegebenheiten ärztlicher Erfahrung versöhnen soll.

Mögen wir auch nicht immer in der glücklichen Lage sein, die von Plato vorgetragenen medizinischen Ansichten bis zu ihren Ursprüngen verfolgen zu können, so ist doch aus den vorstehenden Darlegungen deutlich geworden, daß der athenische Denker der Medizin seiner Zeit ein denkbar großes Interesse entgegengebracht hat. In leidenschaftlichem Erkenntnisdrang hat er sich in jungen Jahren in die Welterklärungsversuche der naturphilosophischen Denker versenkt. Felix qui potuit rerum cognoscere causas: dieses VergiL-Wort hätte als Motto über dem rastlosen Suchen seines jugendlichen Geistes stehen können, als er sich auf die Wanderung durch die Gedankenwelt eines Empedokles und Heraklit, eines Diogenes und Anaxagoras machte ${ }^{79}$. Wenn er auch auf die Dauer kein Genüge fand

77 Vorsokratiker $I^{6}, 219,18 \mathrm{ff}$. (Diels-Kranz).

${ }^{78}$ Philistion, frg. 4 (WellmanN).

${ }^{79}$ Vgl. besonders Phaidon $97 \mathrm{~B}$ ff. 
bei den Antworten, welche die geistigen Führer der Vergangenheit und Gegenwart seinem Fragen darboten, so konnte es doch nicht ausbleiben, daß er damals mit den medizinischen Anschauungen jener Forscher bekannt wurde und sie als dauernden Besitz seinem Gedächtnis einverleibte. Auch in späteren Jahren erlosch sein Interesse an diesen Dingen nicht. Auf seinen Reisen nach Syrakus lernte er den Hofarzt des Dionysios, Philistion von Lokroi, kennen, bei dem er sich über die Erkenntnisfortschritte der sikelischen Ärzte unterrichten konnte. Philistion ist möglicherweise einer Einladung des Oberhauptes der Akademie gefolgt, hat sich in Athen aufgehalten und an dem Logikunterricht Platos teilgenommen. Wenn wir das Bruchstück aus einem zeitgenössischen Lustspiel des EPIKrates auf ihn beziehen und dem Zerrbild des Komikers Glauben schenken dürfen, hat er den Definitions- und Klassifizierungsübungen nicht Geschmack abgewinnen können und manches Mal herzhaft gegähnt ${ }^{80}$. Umgekehrt erwies sich der Philosoph als sehr aufgeschlossen und empfänglich für das, was der Fachmann auf dem Gebiet der Heilkunde ihm an Gedanken und Erkenntnissen zu bieten hatte. Zu Hippokrates vollends schaute Plato voller Verehrung auf. Dessen methodologische Bemühung, in die chaotisch anmutende Vielfalt des Krankheitsgeschehens Ordnung und Übersicht zu bringen durch ein streng nach den Prinzipien der Logik geregeltes Verfahren des Fragens erregte seine lebhafte Anteilnahme und schien ihm weit über die engen Grenzen des medizinischen Fachgebietes für den Betrieb aller Wissenschaft von vorbildlicher Bedeutung zu sein ${ }^{81}$. Eines allerdings vermißte er schmerzlich in der Naturforschung, soweit er sie überschaute. Sie erklärte das Geschehen in der großen und kleinen Welt, in Natur und Mensch, ohne es verständlich zu machen. Ihr Blick war auf das Warum, nicht auf das Wozu gerichtet. Sie suchte der ursächlichen Verkettung der Naturerscheinungen auf die Spur zu kommen und verlor dabei zu leicht die Frage nach Ziel und Bestimmung des bunten Spiels von Entstehen und Vergehen, Wachstum und Verfall aus den Augen. Im Laufe seines langen Lebens hatte sich in Platos Brust mehr und mehr die Überzeugung gefestigt, daß Ordnung und Harmonie, untrügliche Zeugen eines göttlichen Geistes, das Universum durchwalten und auch dem menschlichen Leib, der Wohnstatt der unsterblichen Seele für die Zeit ihres irdischen Wandels, das Siegel göttlichen Ursprungs tief eingeprägt ist. Der Entfaltung und Durch-

${ }^{80}$ Comic. Graec. Fragm. ed. mi. MeInecke, II 682 f. (Kocks Komikerfragmente leider nicht zugänglich).

${ }^{81}$ Phaidros $270 \mathrm{C}$ ff. 
führung dieser Grundanschauung dienen die medizinischen Kapitel des Timaios, in denen das biologische Wissen der Zeit in teleologische Beleuchtung gerückt wird. Die tiefe Gläubigkeit und religiöse Inbrunst, die uns allenthalben aus diesem Werk des Philosophen entgegenstrahlt, hat selten ihren Eindruck auf die Leser alter und neuer Zeit verfehlt. Sie steigert sich zu einem gewaltig brausenden jubelnden Akkord in den Schlußworten, an deren unvergleichlichem Zauber zweitausend Jahre spurlos vorübergerauscht sind: So hat dieses Weltenall sterbliche und unsterbliche Geschöpfe empfangen und ward erfüllt, ein sichtbarer lebendiger Leib, der das Sichtbare in sich birgt, ein wahrnehmbarer Gott und Abglanz des intelligibelen; einzig ist dies Himmelsrund und eingeboren, von riesiger Größe, erlesener Güte, hehrster Schönheit und unendlicher Vollendung.

\section{Literaturverzeichnis}

G. Albert, Die Anschauung des Altertums über die Lehre von der Verdauung, Diss. Würzburg 1912.

E. D. BaumanN, «Praxagoras v. Kos», in Janus 41 (1937) $167 \mathrm{ff}$.

J. Berns, Über das physiologische Denken in den Schriften des Corpus Hippocraticum, Diss. Münster 1944.

G. Bidez, J.-LeboucQ, «Une anatomie antique du cœur humain», in Revue des Etudes Grecques 57 (1944) $7 \mathrm{ff}$.

H. Bonutrau, "Versuch einer kritischen Geschichte der Atmungstheorien», in Archiv für Geschichte der Medizin, 2. Band (1909) $301 \mathrm{ff}$.

A. Castiglioni, A History of Medicine, 2. Auflage, New York 1947.

F. Connford, Platos Cosmology, London 1952.

Сh. Daremberg, «Anatomie et physiologie d'Hérophile», in Revue scientifique 27 (1881) $12 \mathrm{~b}$ ff.

K. Deichgräber und E. Schwyzer, Hippokrates, Über die Entstehung und den Aufbau des menschlichen Körpers, Berlin/Leipzig 1935.

H. Diels, «Über die Excerpte von Menons Iatrika in dem Londoner Papyros 137», in Hermes 28 (1893) $4.07 \mathrm{ff}$.

P. Diepgen, Geschichte der Medizin, 1. Band, Berlin 1949.

H. Diller, Sudhoffs Archiv für Geschichte der Medizin und Naturwissenschaften 29 (1936) $178 \mathrm{ff}$.

H. Diller, Sudhoffs Archiv für Geschichte der Medizin und Naturwissenschaften 31 (1938) $201 \mathrm{ff}$.

J.F. Dobson, «Herophilus of Alexandria», in Proceedings of the Royal Society of Medicine 18 (1924/5), Section of the History of Medicine, $19 \mathrm{ff}$.

C. FREDRICH, Hippokratische Untersuchungen, Berlin 1899.

R. A. Fritzsche, "Der Magnet und die Atmung in antiken Theorien», im Rheinischen Museum 57 (1902) $363 \mathrm{ff}$.

O. Gilbert, Die meteorologischen Theorien des griechischen Altertums, Leipzig 1907. 
E. Hoffmann, in: E. Zeller, Die Philosophie der Griechen II, 15, 1070 ff., Leipzig 1922.

W. JAEgER, Diokles von Karystos, Berlin 1938.

W. JAEger, Nemesios von Emesa, Berlin 1914.

W. JAEger, «Das Pneuma im Lykeion», in Hermes 48 (1913) $29 \mathrm{ff}$.

J.R. LichtenstäDt, Platons Lehre auf dem Gebiet der Naturforschung und der Heilkunde, Leipzig 1826.

Th. Meyer-Steineg, "Studien zur Physiologie des Galenos», in Archiv für Geschichte der Medizin 5 (1912) $172 \mathrm{ff} ., 6$ (1913) $417 \mathrm{ff}$.

E. MüнSAM, «Zur Lehre vom Bau und der Bedeutung des menschlichen Herzens im klassischen Altertum », in Janus 15 (1910) $797 \mathrm{ff}$.

M. Pohlenz, Hippokrates, Berlin 1938.

H. Poschenrieder, Die platonischen Dialoge in ihrem Verhältnis zu den hippokratischen Schriften, Landshut 1882.

F. RüschE, «Blut, Leben und Seele», in Studien zur Geschichte und Kultur des Altertums, 5. Ergänzungsband, Paderborn 1930.

E. SACHS, Die fünf platonischen Körper, Berlin 1917.

J. Schumacher, Antike Medizin I, Berlin 1940.

A.E. TAYLoR, A Commentary on Plato's Timaeus, Oxford 1928.

A.E. Taylor, Plato, 6. Auflage, London 1955.

W. TheILer, Zur Geschichte der teleologischen Naturbetrachtung bis auf Aristoteles, Zürich/ Leipzig 1925.

R. WALZER, «Galens Schrift über die medizinische Erfahrung», in Sitzungsberichte der Preußischen Akademie der Wissenschaften 1932, $449 \mathrm{ff}$.

M. Wellmann, Die Fragmente der sikelischen Ärzte, Berlin 1901.

M. Wellmann, Die pneumatische Ärzteschule, Berlin 1895.

M. WellmanN, «Eine pythagoreische Urkunde des IV. Jahrhunderts v. Chr. », in Hermes 54 (1919) $225 \mathrm{ff}$.

J. Wiberg, «The Medical Science of Ancient Greece: The Doctrine of the Heart», in Janus 41 (1937) $225 \mathrm{ff}$.

U. v. Wilamowitz-Moellendorff, Plato, 2 Bände, Berlin 1919. 